\title{
Estrategias etnográficas para aproximarse al periodismo contemporáneo: propuesta y desafíos
}

\author{
Salvador de León Vázquez ${ }^{1 *}$
}

\begin{abstract}
Resumen examinar críticamente esta y otras propuestas.

Abstract protocols and calling to critically examine this and other proposals.

\section{Palabras Clave}

Etnografía, Metodología cualitativa, Periodismo, Comunicación pública.

Keywords

Ethnography, Qualitative methodology, Journalism, Public communication.

${ }^{1}$ Profesor investigador en la Universidad Autónoma de Aguascalientes.

*Autor para correspondencia: salvador.deleonv@edu.uaa.mx
\end{abstract}

En este ensayo teórico-metodológico se presenta una propuesta para el estudio del periodismo contemporáneo utilizando estrategias etnográficas. El objetivo es el de explicitar algunas de las herramientas utilizadas para conocer la realidad enfocadas al análisis del periodismo, con la finalidad de discutirlas y eventualmente, perfeccionarlas. En el artículo se plantea la necesidad de asumir las transformaciones del periodismo que lo descolocan del ámbito meramente mediático para ganar en territorio analítico aprovechando sus múltiples articulaciones. Se concluye presentando formulaciones para el armado de los protocolos técnicos y llamando a

This text presents a methodological proposal for the study of contemporary journalism using ethnographic strategies. The objective is to make explicit some of the tools used to know the social reality, focused on the analysis of journalism, with the aim of discuss and improve them. The article raises the need to assume the transformations of journalism that displace it from the purely mediatic environment to win in analytical territory taking advantage of its multiple articulations. Conclusions show formulations for the assembly of the technical

\section{El periodismo como práctica y como mundo social}

El periodismo tiene valor como uno de los principales productos culturales para representar el acontecer público ${ }^{1}$. En este ensayo teórico-metodológico se parte del supuesto de que una aproximación a la producción periodística mediante técnicas etnográficas permite la comprensión de sus lógicas y sus prácticas desde el punto de vista de los actores

\footnotetext{
${ }^{1}$ Manuel Martín-Serrano (1994) define acontecer público como "cualquier emergente (cosa, objeto, suceso, observación, idea, norma, relación, etc.) que por su presencia o ausencia afecta o puede afectar a la comunidad y cuyo conocimiento puede ser compartido por los miembros de aquélla, porque ha sido seleccionado como objeto de referencia por las instituciones que tienen a su cargo dar noticia pública de lo que acontece.” (p. 123)
}

sociales. Estas técnicas son la observación participante y la entrevista en profundidad. La aportación será discutir los problemas y desafíos que plantea la época contemporánea, en un contexto de alta tecnologización, dinámicas de desterritorialización-reterritorialización de los actores y los asuntos públicos, cuestionamientos a la centralidad de los medios de comunicación masiva, entre otras cosas, que han significado modificaciones en los discursos, soportes tecnológicos, roles, prácticas y valores del periodismo contemporáneo. En este texto uso la expresión "estrategias etnográficas" para referirme a la forma de investigación social que implica estar allí y hablar con las personas (Geertz, 1989). 
Por tal motivo, el objetivo principal de este ensayo es el de revisar la forma en la que se han utilizado las técnicas etnográficas para comprender el periodismo, poniéndolas en clave de discusión con la intención de contribuir a un diálogo crítico sobre las características de nuestras herramientas metodológicas, sus alcances y limitaciones. La investigación cualitativa aún tiene mucho que aportar, puesto que permite integrar la voz de los actores sociales colocada en contexto, dando la oportunidad de acceder a su mundo de vida, desde su subjetividad, para comprender cómo están experimentando, viviendo y otorgando sentido a los cambios tan veloces que está experimentando el periodismo en todas sus esferas.

Tradicionalmente se ha considerado que los medios de comunicación son el territorio de adscripción del periodismo y el territorio natural para estudiarlo. El enfoque sociológico de la producción noticiosa, de orientación interaccionista, reconoce diferentes niveles de influencia sobre las prácticas periodísticas mismos que se pueden resumir en tres: el primero es el ocupacional o individual en el que se encuentran los valores, creencias, ideologías, formación, aprendizajes, procesos de socialización de cada periodista; el segundo es el organizacional, ámbito propiamente mediático, el de la empresa periodística en donde se establecen rutinas de trabajo, roles en la división del trabajo informativo, criterios organizacionales, líneas de autoridad, producción técnica, soportes tecnológicos, valores noticiosos, etcétera; el tercero es el supraorganizacional, el de la interacción de la empresa periodística con otras empresas del sector mediático, con el Estado, con el mercado, con la sociedad civil, es el marco de la cultura en la que cada empresa periodística está inserta (Hanitzsch, 2007; Hirsch, 1980; Shoemaker y Reese, 1995).

Por otro lado, los estudios de la comunicación pública (Bernier, Watine, Demers, Lavigne, y Moumouni, 2005; Cuadros Rodríguez, Arias García, y Valencia Arias, 2015; De León, 2011; Demers, 2008) demuestran que ampliar el territorio de adscripción del periodismo, de los medios al ámbito de lo público, es fundamental para entender su naturaleza como actividad articuladora de los diversos actores de lo público, funcionando a la vez como profesión, como derecho y libertad civil (a la expresión y a la información), como actividad política, como estrategia mercantil, como dominio tecnológico y como fenómeno cultural, al menos.
En ese sentido, el periodismo como configuración social ${ }^{2}$ exige una mirada amplia que no se circunscriba solamente al ámbito laboral de los periodistas. Implica aproximarse a diversos actores que desarrollan estrategias para obtener visibilidad mediática. Es un campo que está más allá de los datos duros, pues aparece y adquiere sentido y densidad en función de la interacción de los sujetos que participan en él, desde diferentes posiciones, con distintos fines y motivaciones, colocándose en diversas disputas por el dominio del discurso mediático. El periodismo puede verse como un mundo social (Pereira, 2010).

Es necesario revisar la manera en la que se construye conocimiento acerca del periodismo en un momento en el que esta práctica está siendo confrontada por fenómenos complejos como la violencia en contra de los periodistas, la producción y difusión de noticias falsas, la puesta en crisis de los modelos de financiamiento del periodismo contemporáneo, la precarización y flexibilización del trabajo periodístico que coloca en situación de vulnerabilidad económica a quienes ejercen esta profesión, la irrupción de los algoritmos que procesan y arrojan información dirigida a los usuarios de las redes digitales pero, sobre todo, por la renovada toma de conciencia de que es necesario contar con información precisa, plural, y oportuna para fortalecer la calidad de la democracia en nuestras sociedades en transición. Como afirma Voltmer (2013, p. 5) "están surgiendo nuevas formas híbridas de democracia, y con ellas formas híbridas de periodismo, cuyas estructuras y procesos apenas comenzamos a comprender", por lo que es pertinente revisar y discutir las herramientas que usamos para conocer esta realidad.

\section{Estado del arte de los estudios cualitativos del periodismo}

La revisión de literatura que aquí se presenta es muy sintética y comprende tres partes. En primer lugar, los estudios canónicos realizados con un enfoque etnográfico y que constituyeron un modelo inicial de trabajo. En segundo término, la revisión de propuestas metodológicas para el estudio del periodismo. Finalmente, algunas investigaciones

\footnotetext{
${ }^{2}$ La alusión es al concepto de configuración social acuñado por Elias (1990), que corresponde a la figura global y siempre cambiante que forman los participantes de un conjunto de relaciones recíprocas. Para mayor información sobre el uso de este concepto en el estudio de la producción periodística se puede revisar a De León (2012, p. 61-68) y Demers (2008).
} 
actuales realizadas con este tipo de diseños para identificar los desplazamientos temáticos.

Respecto a los estudios canónicos, es posible rastrear el origen de los estudios etnográficos del periodismo hasta los trabajos pioneros de Park (1922) como su libro sobre la prensa y los inmigrantes en Chicago. Otro autor que por esa misma época incursionó de forma importante en el análisis del periodismo fue Lippmann (1922) quien discutió la naturaleza de las noticias como representaciones del mundo en contraposición a la noción de verdad, elevando los debates sobre la realidad y su representación periodística a una discusión epistemológica. Más adelante el conocido artículo de Manning White (1950), en el que explica la lógica de la toma de decisiones de un editor en un periódico de una ciudad mediana de Estados Unidos, definió el gatekeeping como una categoría central de la investigación sobre periodismo y marcó la pauta para el desarrollo de la línea de trabajo conocida como Newsmaking Studies o Sociología del Periodismo.

Bajo esa línea se desarrollaron investigaciones que buscaban caracterizar el periodismo desde las prácticas realizadas por los actores a partir de incursiones etnográficas alrededor de categorías como rutinas de producción noticiosa, procesos de la selección de noticias, procedimientos del tratamiento de la información, roles, valores noticiosos y significado de las noticias. Los trabajos precursores de esta época fueron los elaborados por Sigal (1973), Tuchman (1983), Fishman (1983) y Gans (1980), entre otros. Autores como Hirsch (1980) y Dimmick y Coit (1982) conformaron el modelo de análisis con base en tres esferas: individual, organizacional y supraorganizacional. Posteriormente, Shoemaker y Reese (1995) reformularían el modelo de Hirsch estableciendo las áreas de influencia de la producción noticiosa. Durante las décadas de 1970 y 1980, estos autores generaron las bases de los estudios sociológicos modernos de la producción noticiosa.

Durante la década de 1990, trabajos como los de Berkowitz (1992, 1997), Schudson (1997), Schlesinger (1992), McNair (1998), Breed (1997), Zelizer (1997) y Esser (1998), consolidaron esta línea de trabajo. En México, González-Molina (1986, 1990), Hernández (1995) y Cervantes (1996) comenzaron el desarrollo de los estudios de la producción noticiosa mediante aproximaciones de corte etnográfico.
Con relación a las discusiones metodológicas para el estudio de las prácticas periodísticas, destacan las reflexiones de Tuchman $(1991,2002)$ respecto a los abordajes cualitativos en el estudio de la producción de noticias, donde la autora analiza las tres escuelas principales (la economía política, los estudios organizacionales fenomenológicos y los estudios de los mensajes), así como los diseños metodológicos utilizados. Hjarvard (2014) también ofrece una revisión del uso de técnicas cualitativas desde los estudios clásicos hasta la actualidad. En nuestro país, Cervantes (1994) es quien se ha ocupado de manera más atenta de las reflexiones metodológicas discutiendo la relación entre análisis de contenido y etnografía, las categorías desde las cuales se construyen los ejes analíticos pertinentes (Cervantes, 1995b, 1995a, 1996, 2000), así como estados de la cuestión para distinguir los avances y los desafíos sobre esta línea de investigación (Cervantes, 2005). Publicado en México, pero situado en Argentina, se encuentra el trabajo de Rosenberg (2017), quien ofrece también su experiencia en la investigación etnográfica del periodismo.

En tercer término, las investigaciones desarrolladas en México durante las últimas dos décadas muestran, en un primer movimiento, un desplazamiento del análisis de las prácticas periodísticas a las ciudades medianas del país (De León, 2003, 2011; Del Palacio, 2014; González, 2012; Márquez Ramírez, 2012; Reyna García, 2018), mientras que otro movimiento consiste en una apertura temática a aspectos como la digitalización del periodismo (Castillo, 2014; González, 2014), la violencia contra periodistas (Del Palacio, 2012; González de Bustamante y Relly, 2014; Hughes y Márquez-Ramírez, 2017; Rodelo, 2009), el ejercicio del periodismo bajo esquemas distintos a lo convencional (Castillo, 2018; De León, 2018), las prácticas especializadas del periodismo (Romero, 2016), entre otros. A nivel internacional, el macro proyecto Worlds of Journalism ha apropiado las categorías de los estudios canónicos para trasladarlas a mediciones comparativas entre países buscando las distinciones entre una cultura global del periodismo frente a expresiones locales o regionales (Hanusch y Hanitzsch, 2017). En una revisión reciente, se reconocieron los siguientes temas en investigaciones fuera de México durante los últimos 10 años: conformación de la ideología profesional de los periodistas, el desarrollo del periodismo convergente, 
los procesos de la profesionalización del periodismo, la colocación del periodismo en el contexto de la comunicación pública, la relación del periodismo con el sistema político, la constitución de los roles profesionales, el estudio de las prácticas especializadas del periodismo, y la satisfacción laboral de los periodistas, principalmente (De León, 2018).

\section{La importancia de la aproximación cualitativa al estudio del periodismo}

Ahora hay consenso de que la metodología por sí misma no constituye la base de la cientificidad. Son fundamentales las formulaciones coherentes para dar respuesta a las preguntas planteadas (Tarrés, 2001); tal aserción permite superar la dicotomía maniquea de las metodologías cualitativas y cuantitativas. Sin embargo, sigue siendo necesario establecer los alcances de las técnicas etnográficas para colocarlos en términos de debate. La investigación cualitativa permite reconstruir el significado que el mundo social tiene para las personas y mirar de primera mano cómo ese significado es colocado como movilizador de las acciones que llevan a cabo las personas.

Un ejemplo de esto es el trabajo de Rodelo (2009) en un contexto de alto riesgo por la infiltración de las organizaciones delictivas para el trasiego de estupefacientes frente a la presencia de un Estado débil, en el que la autora pudo captar los motivos por los cuales los periodistas se autocensuraban. El debate de la censura en las noticias suele estar relacionado con el control del Estado sobre la prensa; en este caso, los periodistas no pugnaban para garantizar su libertad de expresión, en ejercicio de esa libertad decidieron autocensurarse como una forma de precaución para salvaguardar su integridad física frente a los cárteles de la droga y la colusión de los cuerpos de seguridad y aparatos de justicia. La autocensura adquirió sentido, en este contexto, como la práctica de un "periodismo precavido". Llegar a este entendimiento fue posible gracias a la aproximación con los sujetos para comprender el sentido de su práctica y la manera en la que ésta se ejercía en su mundo social.

Existen pocos acercamientos etnográficos a las prácticas periodísticas en América Latina. No hay certeza de por qué ocurre así, pero una razón podría ser disciplinar, pues el campo académico de la comunicación en nuestra región se orientó desde la década de 1980 hacia el estudio de la cultura, tomando un auge importante el estudio de las audiencias, en gran parte debido al influyente trabajo de varios investigadores $\mathrm{y}$ pensadores latinoamericanos que pusieron el acento en los procesos de recepción y apropiación de los mensajes de los medios enmarcados en las tramas culturales. En ese sentido, las perspectivas que definían a los medios como aparatos hegemónicos, en el contexto de una teoría social crítica latinoamericana, posiblemente abonaron también para que la producción de mensajes mediáticos como objeto de estudio fuera limitada por identificarse como vehículos de la ideología producida por las clases dominantes, de la cual había que distanciarse en favor de una ciencia crítica (De León, 2008).

La otra razón podría deberse a las dificultades para el ingreso al campo en una apuesta metodológica de este tipo (Cervantes, 2005; Hernández, 2000). Los actores que autorizan el acceso a los escenarios de la producción periodística (empresarios y directivos de los medios y directores de las oficinas de prensa) suelen oponer resistencia para que un investigador permanezca durante mucho tiempo en los espacios; convencerlos exige mucho trabajo de parte del investigador, puesto que esta forma de indagación es necesariamente intrusiva. $\mathrm{Si}$, además, tomamos en cuenta que los proyectos de investigación suelen tener plazos perentorios, resulta complicado para el investigador perder tiempo tratando de ingresar al campo.

Sin embargo, cuando se llega a los porteros correctos, a aquellas personas que forman parte del campo y que ayudan al investigador a ingresar a él y a interpretarlo, la experiencia se vuelve sumamente rica en información e interacciones. Esas relaciones y nexos que se construyen, a la postre se traducen en alianzas y profundidad en el conocimiento del campo, y tienen gran valor para utilizarse después en nuevos proyectos de investigación.

Un ejemplo de esto aparece en una investigación sobre la violencia en contra de periodistas en México (De León, Bravo, y Duarte, 2018). Ante la hostilidad que ha experimentado el gremio en los últimos 15 años, emergieron grupos organizados de periodistas en el país a los cuales interesaba acercarse. Por los protocolos de seguridad de los periodistas, los investigadores no obtenían respuesta positiva para establecer el contacto por ser desconocidos. Fue necesario acudir a 
un contacto anterior, un periodista con quien se habían establecido buenas relaciones y que era reconocido por sus colegas para que generara una recomendación mediante la cual ya fue posible continuar con el estudio.

\section{La interacción al centro}

La calidad de la interacción que el investigador pueda mantener con los sujetos participantes es fundamental. Cuando un investigador ingresa al trabajo de campo, se vuelve parte de él, y los resultados de investigación no dependerán únicamente de lo que los periodistas u otros actores digan y hagan entre sí, sino también de la manera en la que el investigador pueda comunicar los objetivos y la importancia de su tarea a los participantes para ganar aliados.

Los actores de la producción periodística tienen, además, la especificidad de ser analíticos, puesto que su actividad consiste en interpretar a los demás: sus acciones, sus declaraciones, sus silencios, e indagar más allá de lo aparente.

Esa función mediadora es tributaria de un mandato de representatividad según la cual los periodistas se afirman como portadores de una cierta legitimidad social. Ésta les permite observar los acontecimientos, interrogar a los actores, explorar los documentos, analizar y criticar los comportamientos, las decisiones, las declaraciones contundentes así como los silencios cómplices a fin de captar lo público. (Bernier, 2005, p. 17)

El desarrollo de esas habilidades les otorga una capacidad autorreflexiva más densa y crítica de la que podría encontrarse en otros campos de investigación. De ahí que el investigador deberá obtener aptitudes suficientes para aprovechar de la mejor manera esta condición de los actores en favor de la investigación.

Estas interacciones incluyen también incorporarse a la estructura en la que se manifiesta el poder y reconocerla, para escapar de una mirada ingenua. De hecho, la interacción que establece el investigador, por lo menos en un principio, debe basarse en los rituales de poder propios del escenario si se quiere asegurar la permanencia durante el tiempo necesario para el proyecto. Esto tiene varios sobreentendidos.
Un primer sobreentendido es que el investigador no es ignorante de esa estructura, por lo que es importante identificar rápidamente los márgenes de acción para tomar decisiones respecto a las formas de observación. Sin embargo, tampoco se encuentra sometido a ella. La distancia crítica y la vigilancia epistemológica evitará que el investigador de pronto se vea incorporado a una dinámica de la rutina de producción informativa, en menoscabo de sus objetivos de investigación. Existen casos de investigadores que llegan a hacer tareas (redactar noticias, seleccionarlas, incluso hacer labores de reportero) al verse envueltos por las dinámicas y las presiones de la actividad periodística a petición expresa de los sujetos de investigación. Rosenberg (2017), por ejemplo, narra que se le pidió escribir noticias para una sección de un diario argentino en ocasión de falta de personal en la sala de redacción, a lo cual accedió y posteriormente buscó darle sentido en términos de su investigación:

Escribir para Sociedad me permitía posicionarme en el lugar de los periodistas al redactar una nota, conocer de primera mano ciertas reglas de escritura, así como las expectativas del editor sobre el trabajo de una redactora, en este caso, novata. Así mismo, podría ver los resultados del proceso de edición ya que tendría en mis manos la nota tal como la escribí y, al día siguiente, como fue publicada. En este sentido era posible acceder a aquellas normas que naturalmente siguen los periodistas y que, ante mi llegada como redactora ocasional, se verían obligados a hacer explícitas para que mi colaboración resultara fructífera. (Rosenberg, 2017, p. 98)

En una situación de este tipo es necesario mantener claridad sobre el rol que el investigador cumple en el escenario, tanto para sí mismo como para los participantes de la investigación. El debate en este punto sería reflexionar si es válido incursionar en algunas tareas que hacen los periodistas como ejercicio cognoscitivo o si lo adecuado es no intervenir. Mi postura es que el investigador no puede acceder al mundo cotidiano de los actores por realizar tareas de manera ocasional, y es preferible dejar que sean los actores quienes resuelvan los problemas como lo harían si el investigador no estuviera presente, y que posteriormente el investigador 
les pida que narren su vivencia con toda la densidad que implica ser parte "natural" de ese mundo cotidiano al cual el investigador llega, por lo regular, como extranjero.

También hay casos en los que el investigador no es extranjero sino nativo del campo. En su investigación Merchant (2017) ya estaba incorporada previamente como reportera de un periódico de Baja California, México, y aprovecha esa condición para realizar su trabajo de campo en el que identificó las relaciones de cortesía entre reporteros y fuentes informativas. En este caso, el desafío consiste en establecer controles epistemológicos que le permitan al investigador tomar distancia, por un lado, para no obviar asuntos que pueden pasar desapercibidos por estar naturalizados para los nativos $\mathrm{y}$, por otro, para tener una mirada crítica que permita analizar todas las dimensiones del objeto de estudio sin privilegios ni concesiones.

Un segundo sobreentendido es que los representantes del poder en el escenario tienen la facultad de negar el acceso al investigador para observar ciertos aspectos o momentos, como las juntas editoriales o reuniones muy específicas para las tomas de decisión. Aquí es necesario aclarar que esa falta de acceso no debe ser vista como fracaso, sino interpretada como un dato significativo. Posteriormente, ese aspecto o momento podrá ser reconstruido a través de entrevistas o diálogos con los sujetos que participaron en él, incluido el mismo actor que negó el acceso. Es posible, incluso, que la permanencia en el escenario y el respeto a sus reglas le otorgue finalmente la confianza de esos actores y que posteriormente el investigador obtenga ingreso a los lugares antes vedados.

Saber quién tiene el poder de facilitar o bloquear el acceso o quiénes se consideran o son considerados por los demás como poseedores de la autoridad suficiente para garantizar o rechazar el acceso es, sin lugar a dudas, un aspecto fundamental del conocimiento sociológico del campo. (Hammersley y Atkinson, 1994, p. 81)

Un sobreentendido más corresponde a los aspectos y momentos en los que sí está permitido el acceso. En un principio, habrá que sujetarse a estos, pero la incursión, la empatía que el investigador sea capaz de generar con las personas y los datos obtenidos permitirán ampliar eventualmente las condiciones de observación. Por supuesto, también hay escenarios y momentos que se encuentran fuera del control de las autoridades como los espacios públicos en los que existe confluencia de los actores del periodismo como las coberturas de actos, las declaraciones públicas, las entrevistas que los periodistas realizan en la calle, en las que el investigador tendrá la oportunidad de participar sin mayor problema y sin necesidad de más autorización que la de los propios participantes de la interacción observada.

\section{Armar los protocolos}

No existe una única forma en la que los protocolos etnográficos puedan ser elaborados, dependerá de aspectos muy particulares como las preguntas de investigación o los ejes teórico-metodológicos definidos. Por protocolos se entiende aquí la planeación respecto al modo en el que las técnicas de investigación deberán ser instrumentadas para la etapa de trabajo de campo. En función de ese despliegue técnico se obtendrá información significativa que podrá ser elaborada en forma de datos para después contrastarlos con la teoría.

Existe una gran cantidad de manuales metodológicos que lo explican de manera general, pero no es fácil encontrar textos que se refieran específicamente al análisis del periodismo contemporáneo. Algunos consejos que pueden ser útiles en la construcción de estas herramientas metodológicas son los siguientes.

1. El escenario de investigación.

Cuando White (1950) publicó su famoso artículo sobre el gatekeeping mediante el cual explicaba cómo se tomaban las decisiones en la sala de redacción de un diario estadounidense, la unidad de análisis era muy clara: el periódico. Ahí existía un escenario completo en el que se sintetizaba un orden social, prácticas productivas de noticias y roles de trabajo. Posteriormente, la tradición de investigación que de ahí surgió mantuvo su atención en el medio como unidad de análisis y en las tareas homológicas de las fuentes informativas y sus rutinas para garantizar la cobertura noticiosa de sus actividades, haciendo conexiones más o menos explícitas con las condiciones estructurales (Cervantes, 1994; Hjarvard, 2014; Schlesinger, 1992; Tuchman, 1991). 
Sin embargo, conforme se ha ido incrementando la complejidad de la práctica periodística, aunado a sus transformaciones a partir de las innovaciones tecnológicas y la adopción de nuevas lógicas para poder utilizarlas, resulta cada vez más nebuloso identificar el escenario desde el que debe investigarse el periodismo. Aunque el periodismo se exprese en productos mediáticos, su ámbito de acción rebasa lo mediático, teniendo un marco más amplio: el de la comunicación pública, es decir, el de las interacciones de todos los actores del espacio público. Esto implica que, como lo plantean Ringoot y Ruellan (2007), el periodismo trasciende a los periodistas, y en su producción participan múltiples actores de las esferas política, económica y social. Cuando en este texto hemos usado la expresión actores de la producción periodística, no nos referimos únicamente a los periodistas, también a todos los actores sociales que entran en contacto con ellos en la generación de productos periodísticos.

Este rodeo es necesario para hablar del escenario de investigación como el lugar en donde uno se coloca para observar la realidad. La discusión que se introduce aquí tiene la intención de apuntar que el periodismo considerado únicamente como un producto mediático es una mirada limitada, y que hay que hacer un esfuerzo analítico por sacar al periodismo de los medios y colocarlo en el ámbito de la comunicación pública (Demers, 2008).

Aunque esto pueda parecer contrario a los consejos metodológicos que recomiendan acotar antes que ampliar el objeto de estudio, no lo es. Se trata, en primer lugar, de un reconocimiento de la complejidad del periodismo y todas sus articulaciones; en segundo lugar, de asumir decisiones en favor de la pertinencia para avanzar en sus diversas manifestaciones que pasan por la hibridación en múltiples niveles: géneros periodísticos (y las prácticas para producirlos), soportes tecnológicos, profesiones, estrategias, escenarios, actores participantes, prácticas sociales e instituciones generadoras, por lo menos.

Por ejemplo, los periodistas ya no producen únicamente para los medios informativos tradicionales; también hay periodistas generando contenidos para portales en Internet, cibermedios informativos con características diversas, cuentas en plataformas de redes sociodigitales que pueden ser institucionales, personales o de terceros, empresas distribuidoras de información por mensajería digital personalizada, y un largo etcétera.

Así pues, el campo de investigación ya no es tan nítido como antes, y exige del investigador una construcción analítica cada vez más compleja para definir, en términos de su problema de investigación, cuál es el escenario en el que debe insertarse para estudiar algún aspecto del periodismo y sus articulaciones. El escenario tiene que definirse, entonces, ya no de manera física (la sala de redacción, por ejemplo), sino como una configuración social (Elias, 1990) de la cual siguen formando parte los espacios físicos, pero que consiste en la figura formada por todos los actores y sus interacciones. Esto significa que ya no es fija sino dinámica conforme aparecen, desaparecen y se transforman las características de actores, prácticas y soportes tecnológicos, y se escenifica en diferentes lugares físicos y digitales.

Así reflexionado, emergen por sí mismos los elementos que llevan a considerar otras derivaciones de la etnografía, con la finalidad de capturar aspectos que escapan a la etnografía clásica. Por ejemplo, la netnografía o etnografía virtual (Kozinets, 2013) en la que el ámbito de las interacciones se mantiene en línea, como fue el caso del estudio de Castillo (2015) en el que se indagan las influencias que tienen los comentarios de la gente sobre las noticias publicadas en tres medios digitales para la producción de nuevas noticias. Otro ejemplo es la etnografía multisituada (Marcus, 1995), en la que se persigue al objeto de estudio en donde aparezca, en múltiples escenarios o incluso distintas localidades, como lo hicieron Relly y González de Bustamante (2014) al recorrer seis estados mexicanos de la frontera con Estados Unidos para entrevistar en profundidad a 45 periodistas con relación al ejercicio de su profesión en zonas de conflicto. Un trabajo más amplio en ese sentido fue desarrollado por González y Reyna (2019) para realizar 93 entrevistas semiestructuradas a periodistas de 23 estados de México con la finalidad de conocer sus percepciones del riesgo en el que desarrollan su actividad profesional.

2. El protocolo de observación.

Descolocar el periodismo de los medios y recolocarlo en el ámbito de la comunicación pública, como hemos sugerido, plantea retos serios en la ejecución del abordaje empírico. Esto no puede lograrse por puro voluntarismo, hay que construir 
analíticamente ese movimiento mediante un marco conceptual que haga posible pensarlo de esa manera (Demers, 2008).

Así pues, para construir un protocolo de observación bajo estas coordenadas será necesario desanclarse de la nitidez y asumir la complejidad para seguir a la gente, las cosas, las metáforas, las historias, las biografías y los conflictos, en donde quiera que aparezcan, como lo sugiere Marcus (1995), en una suerte de investigación nómada que migre con el objeto de estudio (Ibáñez, 1994), persiguiéndolo para obligarlo a aparecer. La sala de redacción y la ronda por las fuentes acompañando a los reporteros se convierten en uno más de los múltiples escenarios posibles, definidos ya no en función de la actividad de los reporteros únicamente, sino de las relaciones sociales que se buscan dilucidar alrededor de la producción periodística.

Para Marcus (1995), la etnografía multisituada se diseña "alrededor de cadenas, patrones, hilos, conjunciones o yuxtaposiciones de lugares" (pág. 105), lo cual es perfectamente compatible con el análisis de las prácticas de los actores del periodismo. Definidas estas yuxtaposiciones en las que el periodismo se escenifica, habría que establecer analíticamente las categorías que formarán parte del protocolo de observación, las cuales probablemente estarán dadas en función de los actores y sus prácticas.

De manera mínima, siguiendo los preceptos de la etnografía, los elementos colocados en la tabla anterior deberán ser desarrollados durante el trabajo de campo, haciéndose específicos en función de la construcción del objeto de estudio y la definición de sus ejes. El instrumento que ayuda en el registro son las notas de campo. Algunos investigadores optan por usar una ficha de observación en donde se organizan a priori los observables, lo cual tiene la ventaja de organizar la observación desde su realización, cuidando de mantener siempre la opción de anotaciones extras sobre asuntos no previstos pero que resultarán relevantes. El acceso al campo empírico de investigación es negociable $\mathrm{y}$, de hecho, negociado. Para pasar por esta transacción no existe una única manera; lo que funcionó en una investigación puede no ser lo adecuado en otra, pero hay estrategias que resulta útil recuperar de experiencias previas en medios periodísticos.

Cuando las relaciones a observar involucran a periodistas enfrentan un obstáculo en común: estos profesionales disponen de poco tiempo libre, trabajan bajo presión y sin un horario fijo, particularmente quienes tienen condiciones precarias y no cuentan con un contrato formal (como los llamados freelancer), se les paga por trabajo publicado, o cubren fuentes informativas con actividades muy dispersas. Conforme se ha ido precarizando y flexibilizando la labor periodística a partir de tareas que antes estaban distribuidas en varias personas y que ahora hace una sola por el mismo sueldo, a veces por uno menor, el ritmo de trabajo se ha vuelto más frenético que antes. Entre las tareas que ahora asumen están el redactar para los soportes escritos como el impreso y el digital, producir imagen fija y en movimiento, producir audio, hacer la postproducción e integración de la información para la difusión multiplataforma, generar los mensajes escritos y/o audiovisuales para redes sociodigitales, etc. Por lo general, los periodistas acostumbran trabajar sin interrupciones y valoran los espacios de libertad. En esa vorágine que los envuelve, lograr que atiendan y entiendan las peticiones del investigador es el primer reto.

Una vez superado, el campo manda. Es decir, las condiciones de la observación son las que las dinámicas de los actores establecen, y el investigador deberá estar dispuesto a incorporarse a ellas en aras de mantener la pertinencia. Gran parte de las veces, la complejidad del campo rebasa las capacidades del responsable de la investigación para atender todas las aristas que se despliegan, por lo que es necesario tomar decisiones estratégicas que permitan registrar las prácticas realizadas por los actores. Por ejemplo:

En las tres áreas [Coordinación de Tiempo Real; Edición; Coordinación de Agencia] la etapa inicial de campo se basó en charlas in situ con los periodistas y la observación participante de las interacciones entre ellos, que ayudaron a identificar la vida cotidiana dentro de la organización para cada equipo de trabajo y para el grupo en general: afectos, opiniones, roles, jerarquías y desacuerdos. Durante esta fase permanecer con los periodistas durante toda su jornada, especialmente los fines de semana o por las tardes, fue una herramienta valiosa para generar rapport.

En la etapa intermedia, la atención se centró en los aspectos operativos de cada área y su relación 
Tabla 1. Elementos del protocolo de observación

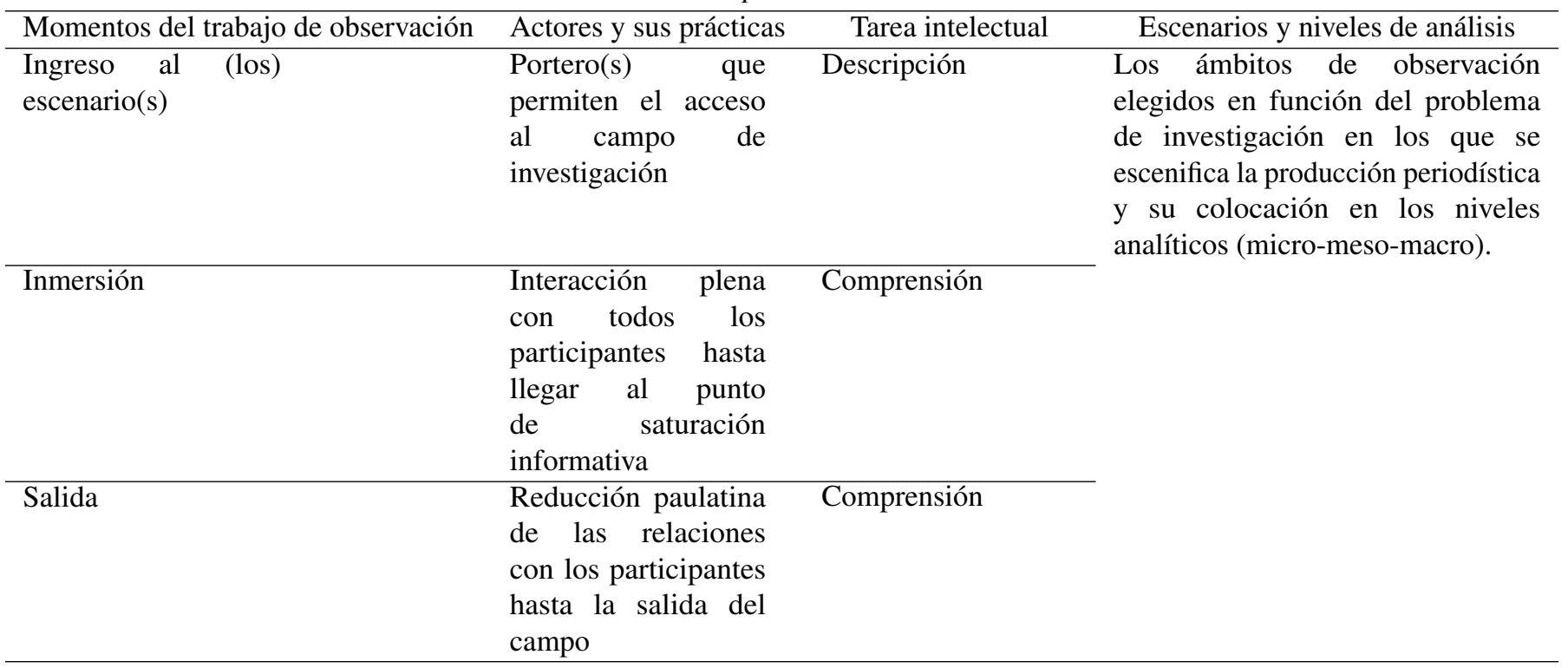

Fuente: elaboración propia.

con los demás; en la toma de decisiones y los factores que influían en ellas.

Debido a la dispersión de las rutinas de los reporteros que trabajan fuera de las oficinas $y$ a la naturaleza de sus fuentes, durante esta etapa únicamente fue posible acompañar al de la fuente legislativa en sus jornadas de trabajo. Las del resto se reconstruyeron a partir de lo observado y de lo que ellos y/o sus compañeros aportaron en entrevistas y pláticas casuales. (González, 2014, p. 95)

En la referencia anterior se identifica que, en una primera instancia, la investigadora se esfuerza por mantener interacciones con la mayor parte del equipo de periodistas de este cibermedio y obedecer así al principio implícito de planear sus incursiones en función de las demandas que le plantea el propio campo de investigación. Sin embargo, de manera posterior tiene que tomar la decisión de delimitar su participación y recuperar las experiencias no observadas directamente mediante otras estrategias de obtención de datos. Es aquí donde se hace patente el apuntalamiento de la otra técnica que forma parte de los acercamientos etnográficos, la entrevista.
3. El protocolo de la entrevista.

La entrevista en profundidad es la contraparte de la observación. La propia incursión en el trabajo de campo y las interacciones derivadas de este, permitirán identificar a los participantes idóneos para ser entrevistados y los momentos adecuados. Su función consiste en dejar hablar a los actores sobre sus prácticas "en ese sentido, el lenguaje, más que un medio de comunicación, se concibe como un instrumento de conocimiento cultural" (Vela, 2001, p. 73). Los discursos que despliegan los actores sobre su mundo de vida, nos dan acceso a él y a los significados que guarda para quienes actúan ahí:

La entrevista es el lugar y el tiempo de encuentro entre el sujeto investigador y el sujeto social no profesional; ahí se intercambia la información, se teje la urdimbre del proceso de conocimiento etnográfico. A partir de la entrevista se descubre y analiza el mundo social obviado en la vida cotidiana; la entrevista abre la vida ordinaria, pues en la situación de entrevista el mundo social se pone en duda, se construye como objeto de estudio. (Galindo, 2010, p. 264)

Los actores de la producción periodística, particularmente, tienen la peculiaridad de encontrarse muy familiarizados con 
Tabla 2. Ejemplo de guía de entrevista sobre la producción de periodismo político.

\begin{tabular}{|c|c|}
\hline Tema & Objetivo \\
\hline Trayectoria & $\begin{array}{l}\text { Identificar la biografía del entrevistado, cómo fue } \\
\text { construyendo su propia trayectoria que lo lleva al } \\
\text { lugar en el que hoy se encuentra, con el objetivo } \\
\text { de situar en el tiempo y en el espacio su práctica } \\
\text { periodística o política. }\end{array}$ \\
\hline Percepción de los medios informativos & $\begin{array}{l}\text { Conocer qué percepción tiene del trabajo } \\
\text { periodístico que ejercen los medios informativos } \\
\text { en general y en la localidad, como cree que debería } \\
\text { ser ese trabajo, cómo es en realidad, qué opinión } \\
\text { tiene de la forma en que los propietarios de los } \\
\text { medios ejercen esa propiedad. }\end{array}$ \\
\hline Relación con los medios informativos & $\begin{array}{l}\text { Definir cómo es la relación que el entrevistado } \\
\text { mantiene con los medios informativos locales, } \\
\text { regionales y nacionales y con sus representantes } \\
\text { (los periodistas), cómo usa el periodismo (en el } \\
\text { sentido de construir prácticas y estrategias). }\end{array}$ \\
\hline Situaciones concretas & $\begin{array}{l}\text { Usar la información de los temas anteriores como } \\
\text { insumo para preguntar sobre situaciones concretas } \\
\text { en las que el entrevistado ha tenido o tiene relación } \\
\text { con el periodismo, así como de problemáticas } \\
\text { directas que han aparecido durante el periodo del } \\
\text { trabajo de campo, con la finalidad de profundizar } \\
\text { de manera situada en los datos. }\end{array}$ \\
\hline
\end{tabular}

Duración aproximada de la entrevista: 1.5 a 2 horas.

Fuente: De León (2012, p. 208).

la realización de entrevistas; quizás resulte una obviedad decirlo si consideramos que es un insumo básico para la elaboración de productos informativos. Sin embargo, es necesario tenerlo en cuenta debido al sentido que cobran los roles y los dispositivos de las entrevistas para los actores de la producción periodística, puesto que representa un desafío metodológico: los periodistas se asumen como entrevistadores; ellos son quienes acostumbran otorgar la palabra y definir los temas de la comunicación. Más aún, la grabadora o los nuevos dispositivos para el registro de la voz y la imagen son un símbolo de la función y el poder del periodista, son parte de su identidad. Estos dispositivos, al registrar, pone en situación vulnerable a quien ofrece testimonio y otorga poder a su portador. De manera que no es raro que constantemente se le pida al entrevistador que detenga la grabación para expresar situaciones que consideran delicadas, y que prefieren revelar sin que exista registro. Este asunto, desde mi punto de vista, no ha sido lo suficientemente problematizado por los investigadores del periodismo en las discusiones metodológicas.

Es por este simbolismo que guarda la entrevista en el mundo periodístico por lo que ha de mantenerse una cautela especial en la realización de las entrevistas etnográficas. Se sugiere aplicar esta técnica una vez que el investigador haya tenido la oportunidad de familiarizarse con el campo, lo que se traduce en reconocer a los actores, dejarse conocer por ellos y haber generado la empatía suficiente para colocarlos delante de la grabadora sin recelos.

Una vez atendido esto, la entrevista consiste en dejar que la persona se exprese libremente, guiado de forma sutil por el investigador, evitando cuestionarios rígidos en favor de guías generales de temas de conversación para atender el objeto de estudio. A continuación se ofrecen algunos ejemplos de guías de entrevista usados en investigaciones reales sobre la producción periodística. 
Tabla 3. Ejemplo de guía de entrevista sobre criterios de noticiabilidad en un cibermedio.

\begin{tabular}{|c|c|}
\hline Temas de entrevista & Informantes \\
\hline 1) Qué son y cómo se hacen las noticias. & $\begin{array}{l}\text { Directivos o jefes (altos mandos): directores y } \\
\text { subdirectores de las unidades de análisis. }\end{array}$ \\
\hline 2) Las fuentes y los temas de las noticias. & \\
\hline 3) Qué es o cómo es el periodismo actualmente. & \\
\hline 4) El rol o la función social del periodista. & $\begin{array}{l}\text { Coordinadores y staff (mandos medios y } \\
\text { subordinados): reporteros, encargados de sección } \\
\text { y coeditores. }\end{array}$ \\
\hline
\end{tabular}

Fuente: González (2014, p. 87).

Tabla 4. Ejemplo de guía de entrevista a corresponsales periodísticos en el caso Ayotzinapa.

\begin{tabular}{|c|c|}
\hline Primera fase & Segunda fase \\
\hline Definir los perfiles de los participantes. & Conocer la rutina de producción de noticias. \\
\hline $\begin{array}{l}\text { Comprobar su relación con la organización } \\
\text { informativa. }\end{array}$ & $\begin{array}{l}\text { Comprender los cambios en el proceso de } \\
\text { recolección de noticias suscitados a partir del caso } \\
\text { Ayotzinapa. }\end{array}$ \\
\hline $\begin{array}{l}\text { Reconocer la trayectoria del corresponsal de } \\
\text { prensa, las opiniones sobre el contexto de violencia } \\
\text { y en particular el interés del periodista por informar } \\
\text { sobre Ayotzinapa. }\end{array}$ & $\begin{array}{l}\text { Identificar los procesos de negociación y las } \\
\text { condicionantes organizacionales que median el } \\
\text { proceso de construcción de la noticias. }\end{array}$ \\
\hline & $\begin{array}{l}\text { Precisar las estrategias y medidas que emplearon } \\
\text { los periodistas para realizar la cobertura en el } \\
\text { contexto de violencia. }\end{array}$ \\
\hline
\end{tabular}

Fuente: elaboración propia a partir de la estrategia metodológica empleada por Castillo. (2018, p. 54)

Como puede observarse en los tres ejemplos provistos, las guías de entrevista consisten en un número limitado de tópicos de conversación surgidos de los objetivos de la investigación. Recorriendo esos tópicos, no necesariamente en el orden inscrito, el investigador guía el diálogo para cumplir con la tarea de descubrir y analizar el mundo social obviado en la vida cotidiana que nos propone Galindo (2010).

\section{Discusión final}

Las estrategias metodológicas que usamos para estudiar el periodismo bajo los rasgos que lo definen en la actualidad, merecen ser reflexionadas y discutidas con la finalidad de clarificar su utilidad y validez. En este caso, hemos puesto bajo examen las condiciones bajo las cuales es posible acercarse al estudio de la producción periodística mediante una orientación etnográfica. Este texto pretende ser una propuesta, nunca terminada, sino puesta en la mesa de discusión para avanzar en la afinación de las herramientas a nuestra disposición.

Los rápidos cambios que experimenta la profesión periodística y las relaciones sociales que de ella se desprenden exigen volver constantemente sobre nuestros pasos en el proceso de generar conocimiento para revisarlos $\mathrm{y}$ verificar su pertinencia, de manera que los objetos sociales puedan ser conocidos en forma empírica, con el máximo rigor, identificando las inflexiones, las transformaciones, las adiciones, los embates y las nuevas localizaciones que el periodismo incorpora en tanto que constituye un fenómeno social complejo.

Es por eso que, por lo menos, en una aproximación etnográfica del periodismo deben considerarse su ampliación de lo mediático hacia lo público, sus transformaciones tecnológicas que no solamente son técnicas sino también políticas y culturales, las prácticas reales de los actores de 
su producción para definirlo a partir de ellas y no desde una perspectiva esencialista e ideal. Eso nos permitiría reconstruirlo teóricamente con la pertinencia adecuada.

En este texto se ha propuesto que el marco teórico de la comunicación pública puede ofrecer un punto de partida para realizar esos movimientos analíticos. También se han señalado algunas características que tiene el ámbito de la producción periodística como campo de investigación específico para plantear algunos aspectos que son necesarios tener en cuenta cuando se ingresa a él como investigador.

Además, se han ofrecido algunas ideas sobre los protocolos técnicos correspondientes a la observación participante y la entrevista como técnicas fundamentales de las aproximaciones etnográficas, considerando las especificidades de este campo de investigación.

Lo presentado aquí es una propuesta y, como toda propuesta, no pretende ser normativa, antes que nada, busca aportar elementos para hacer explícito y discutible cómo conocemos la realidad, y en esa medida, evaluar la validez y pertinencia de ese conocimiento.

\section{Referencias}

Berkowitz, D. (1992). Non-Routine News and Newswork: Exploring a What-a-Story. Journal of Communication, 42(1), 82-94. https://doi.org/10.1111/j.1460-2466.1992 .tb00770.x

Berkowitz, D. A. (1997). Social meanings of news: a text-reader. USA: Sage.

Bernier, M.-F. (2005). Pourquoi les journalistes? Les contours d'un ideal journalistique. En M.-F. Bernier, T. Watine, F. Demers, A. Lavigne, y C. Moumouni (Eds.), Pratiques novatrices en communication publique. Journalisme, relations publiques et publicité (pp. 13-42). Quebec: Université Laval.

Bernier, M.-F., Watine, T., Demers, F., Lavigne, A., y Moumouni, C. (2005). Practiques novatrices en communication publique. Quebec: Les presses de l'Université Laval.

Breed, W. (1997). Social control in the newsroom: a functional analysis. En D. A. Berkowitz (Ed.), Social meanings of news: a text-reader. USA: Sage.

Castillo, D. (2018). La producción periodística del Caso Ayotzinapa: un estudio sobre las rutinas de producción informativa de los corresponsales de prensa de La Jornada y El Universal. Maestría en Cultura y Comunicación. Universidad Veracruzana.
Castillo, L. (2014). Interactividad y audiencias en los periódicos digitales. Estudio de tres medios mexicanos. Intersticios Sociales, 1-25.

Castillo, L. (2015). La interactividad en el periodismo digital mexicano: las participaciones de las audiencias. El estudio de tres casos: El Diario.mx, El Universal y Sinembargo.mx. Doctorado en Investigación. El Colegio de Chihuahua.

Cervantes, C. (1994). Análisis de contenido y etnografía en el estudio de la producción de noticias. In E. E. Sánchez-Ruiz \& C. Cervantes (Eds.), Investigar la comunicación. Propuestas iberoamericanas (pp. 77-104). México: ALAIC, Universidad de Guadalajara.

Cervantes, C. (1995a). ¿De qué se constituye el habitus en la práctica periodística? Comunicación y Sociedad, (24), 97-125. Recuperado de http://publicaciones.cucsh.udg. mx/pperiod/comsoc/pdf/24_1995/97-125.pdf

Cervantes, C. (1995b). Valores noticiosos en el periodismo televisivo de nota roja. Búsqueda articulada de indicadores empíricos. Comunicación y Sociedad, (25), 89-137. Recuperado de http://www.publicaciones.cucs h.udg.mx/pperiod/comsoc/pdf/25-26_1996/89-137.pdf

Cervantes, C. (1996). Construcción primaria del acontecer y planeación de la cobertura informativa. Propuesta metodológica para su estudio. Comunicación y Sociedad, (28), 49-81. Recuperado de http://www.publicaciones. cucsh.udg.mx/pperiod/comsoc/pdf/28_1996/49-81.pdf

Cervantes, C. (2000). Para superar la ruta de modelos, efectos y metáforas equívocas en la sociología del periodismo. En G. Orozco (Ed.), Lo viejo y lo nuevo. Investigar la comunicación en el siglo XXI. Madrid: De la Torre.

Cervantes, C. (2005). El estudio de los productores de noticias: desarrollo internacional y avances de investigación en México. En J. C. Lozano (Ed.), La comunicación en México: Diagnósticos, balances y retos (pp. 91-132). México: CONEICC.

Cuadros Rodríguez, J. A., Arias García, S., y Valencia Arias, A. (2015). La comunicación pública como estrategia orientadora en los procesos de participación ciudadana de los jóvenes. Revista Encuentros, 13(1), 111-122. http s://doi.org/10.15665/re.v13i1.353

De León, S. (2003). La construcción del acontecer. Análisis de las prácticas periodísticas. México: CONEICC, Universidad de Guadalajara, Universidad Autónoma de Aguascalientes.

De León, S. (2008). Notas para una exploración teórica sobre los estudios de producción de comunicación mediática (periodismo, opinión pública y comunicación política). Comunicación y Sociedad, 9, 145-173.

De León, S. (2011). Comunicación pública, transición política y periodismo en México: el caso de Aguascalientes. Comunicación y Sociedad, 15, 43-69.

De León, S. (2012). Comunicación pública y transición política. Los rasgos de lo global en el periodismo local. 
Un estudio situado. México: Universidad Autónoma de Aguascalientes.

De León, S. (2018). Una mirada a las rutinas no convencionales de producción periodística en México. En M. E. Hernández (Ed.), Estudios sobre periodismo en México: despegue e institucionalización (pp. 149-170). México: Universidad de Guadalajara.

De León, S., Bravo, A., y Duarte, E. M. (2018). Entre abrazos y golpes... Estrategias subpolíticas de periodistas mexicanos frente al riesgo. Sur Le Journalisme, 7, 114-129.

Del Palacio, C. (2014). Labores de ritualización y mitificación en las secciones policiacas de tres periódicos de Veracruz a través de sus imágenes. Relaciones, (140), 71-106.

Del Palacio Montiel, C. (2012). En Veracruz se aprende a vivir con miedo: la Construcción social de la violencia a través de los periódicos de Veracruz en México (2005-2011). Comunicação \& Informação, 15(1), 32-45.

Demers, F. (2008). La communication publique, un concept pour repositionner le journalisme contemporain. Les Cahiers Du Journalisme, 18, 208-230. Retrieved from http://www.cahiersdujournalisme.net/pdf/18/13_DEM ERS.pdf

Demers, F., y Lavigne, A. (2007). La comunicación pública: una prioridad contemporánea de investigación. Comunicación y Sociedad, (7), 65-87.

Dimmick, J., y Coit, P. (1982). Levels of analysis in mass media decision making. A taxonomy research strategy and ilustrative data analysis. Communication Research, 9(1), 3-32. https://doi.org/10.1177/0093650820090010 01

Elias, N. (1990). La sociedad de los individuos. Barcelona: Península.

Esser, F. (1998). Editorial Structures and Work Principles in British and German Newsrooms. European Journal of Communication, 13(3), 375-405. https://doi.org/10.117 7/0267323198013003004

Fishman, M. (1983). La fabricación de la noticia. Buenos Aires: Tres Tiempos.

Galindo, J. (2010). Encuentro de subjetividades. Objetividad descubierta. La entrevista como centro de trabajo etnográfico. In K. Covarrubias, L. I. Rodríguez, y G. Zenteno (Eds.), El recurso de la metodología: cultura y sociedades complejas (pp. 257-318). México: Universidad de Colima.

Gans, H. (1980). Deciding what's news. A study of CBS News, NBC Nightly News, Newsweek and Time. New York: Vintage Books.

Geertz, C. (1989). El antropólogo como autor. Barcelona: Paidós.

González-Molina, G. (1986). Valores noticiosos: la distribución desigual del acceso periodístico. México: Universidad de Colima.
González-Molina, G. (1990). The production of mexican television news. The supremacy of corporate rationale. Leicester.

González de Bustamante, C., y E. Relly, J. (2014). Journalism in times of violence: Social media use by US and Mexican journalists working in northern Mexico. Digital Journalism, 2(4), 507-523. https://doi.org/10.1080/2167 0811.2014 .882067

González, J. C. (2014). Construcción social de la noticia: negociación de los criterios de noticiabilidad en el cibermedio mexicano. Maestría en Investigaciones Sociales y Humanísticas. Universidad Autónoma de Aguascalientes.

González, R. A. (2012). Change and continuity in Mexican Journalism. The University of Leeds.

González, R. A., y Reyna, V. H. (2019). "They don’t trust us; they don't care if we're attacked": trust and risk perception in Mexican journalism. Communication \& Society, 32(1), 147-160. https://doi.org/10.15581/003.3 2.1.147-160

Hammersley, M., y Atkinson, P. (1994). Etnografía. Métodos de investigación. Barcelona: Paidós.

Hanitzsch, T. (2007). Deconstructing journalism culture: Toward a universal theory. Communication Theory, 17(4), 367-385. https://doi.org/10.1111/j.1468-288 5.2007.00303.x

Hanusch, F., y Hanitzsch, T. (2017). Comparing Journalistic Cultures Across Nations: What we can learn from the Worlds of Journalism Study. Journalism Studies, 18(5). https://doi.org/10.1080/1461670X.2017.1280229

Hernández, M. E. (1995). La producción noticiosa. México: Universidad de Guadalajara.

Hernández, M. E. (2000). La investigación sobre producción de noticias desde América Latina. En G. Orozco (Ed.), Lo viejo y lo nuevo. Investigar la comunicación en el siglo XXI (pp. 155-168). España: Ediciones De La Torre.

Hirsch, P. (1980). Occupational, organizational and institutional models in mass media research. Toward and Integrated Framework. Mass Communication Review Yearbook, 1, 265-294.

Hjarvard, S. (2014). El estudio de la producción de noticias. En K. B. Jensen (Ed.), La comunicación y los medios. Metodologías de investigación cualitativa y cuantitativa (pp. 145-172). México: Fondo de Cultura Económica.

Hughes, S., y Márquez-Ramírez, M. (2017). Examining the practices that Mexican journalists employ to reduce risk in a context of violence. International Journal of Communication, 11(January), 499-521.

Ibáñez, J. (1994). El regreso al sujeto. La investigación social de segundo orden. España: Siglo XXI.

Kozinets, R. (2013).Netnography: Redefined. London. https: //doi.org/10.1002/9781118767771.wbiedcs067

Lippmann, W. (1922). Public opinion. New York: Harcourt, Brace and Company. 
Marcus, G. (1995). Ethnography in/of the world system: the emergent of multi-sited ethnography. Annual Review of Anthropology, (27), 95-117.

Márquez Ramírez, M. (2012). Change or Continuity: The Culture and Practices of Journalism in Mexico (2000-2007). Recuperado de http://eprints.gold.ac. uk/8011/

Martín-Serrano, M. (1994). La producción social de comunicación. México: Alianza Editorial.

McNair, B. (1998). Sociology of journalism. London: Arnold.

Merchant, D. (2017). Relaciones de cortesía en el campo periodístico de Baja California, México: El caso de los periodistas de periódicos y oficinas de comunicación social. Comhumanitas, 8(1), 81-102.

Park, R. E. (1922). The immigrant press and its control. Americanization studies. New York: Harper \& Bros. Recuperado de http://nrs.harvard.edu/urn-3:FHCL: 1186546

Pereira, F. H. (2010). El mundo de los periodistas: aspectos teóricos y metodológicos. Comunicación y Sociedad, $101-124$.

Relly, J. E., y González de Bustamante, C. (2014). Silencing Mexico: A Study of Influences on Journalists in the Northern States. International Journal of Press/Politics, 19(1), 108-131. https://doi.org/10.1177/194016121350 9285

Reyna García, V. H. (2018). Objetividad y conteo de cuerpos en el periodismo sonorense. Revista Mexicana de Ciencias Políticas y Sociales, 63(233), 93-116. https: //doi.org/10.22201/fcpys.2448492xe.2018.233.58859

Rodelo Amezcua, F. V. (2009). Periodismo en entornos violentos: el caso de los periodistas de Culiacán, Sinaloa. Comunicación y Sociedad, (12), 101-118.

Romero, L. (2016). Producción informativa en salud: periodismo radiofónico en Puebla. Disertaciones. Anuario electrónico estudios de comunicación social (Vol. 9). https://doi.org/10.12804/disertaciones.09.01.2 016.07

Rosenberg, L. (2017). Apuntes sobre la inserción del investigador en la sala de redacción de un diario argentino 1, 87-109.

Ruellan, D., y Ringoot, R. (2007). Journalism as permanent and collective invention. Brazilian Journalism Research, 3(2), 67-76.

Schlesinger, P. (1992). Repensando la sociología del periodismo: estrategias de las fuentes y límites del centralismo en los medios. Estudios Sobre Las Culturas Contemporáneas, IV (14), 279-307. Recuperado de https://www.redalyc.org/articulo.oa?id=31641416

Schudson, M. (1997). The sociology of news production. En D. A. Berkowitz (Ed.), Social meanings of news: a text-reader (pp. 7-22). USA: Sage.
Shoemaker, P. J., y Reese, S. D. (1995). Mediating the Message: Theories of Influence on Mass Media Content. USA: Longman.

Sigal, L. (1973). Reporteros y funcionarios. La organización y las normas de la elaboración de noticias. México: Gernika.

Tarrés, M. L. (2001). Observar, escuchar y comprender. Sobre la tradición cualitativa en la investigación social. México: Miguel Ángel Porrúa.

Tuchman, G. (1983). La producción de la noticia. Barcelona: Gustavo Gili.

Tuchman, G. (1991). Qualitative methods in the study of news. En K. B. Jensen \& N. W. Jankowsky (Eds.), A handbook of qualitative methodologies for mass communication research (pp. 79-92). London: Routledge.

Tuchman, G. (2002). The production of news. En K. B. Jensen (Ed.), A handbook of media and communication research: qualitative and quantitative methodologies (pp. 78-90). USA: Routledge.

Vela, F. (2001). Un acto metodológico básico de la investigación social: la entrevista cualitativa. En M. L. Tarrés (Ed.), Observar, escuchar y comprender. Sobre la tradición cualitativa en la investigación social (pp. 65-96). México: Miguel Ángel Porrúa.

Voltmer, K. (2013). The media in transitional democracies. Cambridge: Polity Press.

White, M. (1950). The "gate keeper": a case study in the selection of news. Journalism Quarterly, (27), 383-390.

Zelizer, B. (1997). Journalists as interpretative communities. En D. A. Berkowitz (Ed.), Social meanings of news: a text-reader. USA: Sage. 